\title{
Typologie des liens hypertextuels dans les journaux électroniques
}

\section{The Typology of Hypertext Links of Electronic Newspapers Tipología de los enlaces hipertextuales en los periódicos electrónicos}

\author{
Johanne Charbonneau
}

Volume 45, numéro 1, janvier-mars 1999

URI : https://id.erudit.org/iderudit/1032773ar

DOI : https://doi.org/10.7202/1032773ar

Aller au sommaire du numéro

\section{Éditeur(s)}

Association pour l'avancement des sciences et des techniques de la documentation (ASTED)

\section{ISSN}

0315-2340 (imprimé)

2291-8949 (numérique)

Découvrir la revue

Citer cet article

Charbonneau, J. (1999). Typologie des liens hypertextuels dans les journaux électroniques. Documentation et bibliothèques, 45(1), 7-14.

https://doi.org/10.7202/1032773ar

\section{Résumé de l'article}

Cette recherche avait pour but de valider une typologie établie pour classer les types de liens hypertextuels utilisés par les journaux électroniques. Cette typologie a été appliquée à un échantillon de 170 liens tirés de divers journaux. Celle-ci comprend deux grands thèmes : le premier, la description conceptuelle qui couvre le sens (sémantique, rhétorique) et l'utilité du lien et le deuxième, la description physique qui analyse la direction (interne, externe) et le support vers lequel il dirige (texte, image, etc.). Ces types se divisent en sous-catégories pour mieux définir chacun des liens. Les résultats valident la typologie et son application à d'autres échantillons de liens hypertextuels. On note une forte tendance des journaux à privilégier les liens sémantiques menant, le plus souvent, à un texte ou à des sources provenant du même journal.
Tous droits réservés ( $)$ Association pour l'avancement des sciences et des techniques de la documentation (ASTED), 1999
Ce document est protégé par la loi sur le droit d'auteur. L'utilisation des services d'Érudit (y compris la reproduction) est assujettie à sa politique d'utilisation que vous pouvez consulter en ligne.

https://apropos.erudit.org/fr/usagers/politique-dutilisation/ 
Le Prix Paul-Aimé Martin a été créé, en 1991, par l'ASTED, en collaboration avec les Services documentaires multimédia (SDM). Le but de ce prix est:

- de rendre hommage au révérend père Paul-Aimé Martin, c.s.c., qui a poursuivi durant plus d'un demi-siècle une carrière entièrement consacrée à la promotion du livre, de la lecture et des bibliothèques. Fondateur et président-directeur général des Éditions Fides durant plusieurs décennies, il fut aussi un des fondateurs de l'École des bibliothécaires qui deviendra l'École de bibliothéconomie au moment où elle sera affiliée à l'Université de Montréal. II sera aussi un des fondateurs de la première association francophone de bibliothécaires canadiens, l'Association canadienne des bibliothèques d'institutions (ACBI), en 1944; dienne.

m et de signaler publiquement le travail de recherche d'une étudiante ou d'un étudiant d'une école de bibliothéconomie cana-

La lauréate de cette année est Johanne Charbonneau de l'École de bibliothéconomie et des sciences de l'information de l'Université de Montréal. Le prix comporte une bourse de $500 \$$ et la publication de son texte dans cette revue.

\title{
Typologie des liens hypertextuels dans les journaux électroniques
}

\author{
Johanne Charbonneau
}

Cette recherche avait pour but de valider une typologie établie pour classer les types de liens hypertextuels utilisés par les journaux électroniques. Cette typologie a été appliquée à un échantillon de 170 liens tirés de divers journaux. Celle-ci comprend deux grands thèmes: le premier, la description conceptuelle qui couvre le sens (sémantique, rhétorique) et l'utilité du lien et le deuxième, la description physique qui analyse la direction (interne, externe) et le support vers lequel il dirige (texte, image, etc.). Ces types se divisent en souscatégories pour mieux définir chacun des liens. Les résultats valident la typologie et son application à d'autres échantillons de liens hypertextuels. On note une forte tendance des journaux à privilégier les liens sémantiques menant, le plus souvent, à un texte ou à des sources provenant du même journal.

The Typology of Hypertext Links of Electronic Newspapers In order to validate the typology used to classify the types of hypertext links used in electronic newpapers, it was applied to a sample of 170 links used in different newspapers. This typology includes two themes : a conceptual description that covers the meaning (semantic, rhetoric) and the use of the link and, secondly, the physical description that analyses the direction (internal or external) and the support it seems to favour (text, image, etc.). These types are further divided into sub-categories that help define each link. The results validate the typology and its application to other hypertext link samples. A strong tendancy was observed among newspapers to prefer sematic links leading, more often than not, to a text or to sources from the same journal.

\section{Tipología de los enlaces hipertextuales en los periódicos electrónicos}

Con el fin de convalidar una tipología establecida para clasificar los enlaces hipertextuales utilizados por los periódicos electrónicos, dicha tipología se aplicó a una muestra de 170 enlaces extraídos de diversos periódicos. Esta tipología comprende dos grandes temas: el primero se refiere a la descripción conceptual que cubre el sentido (semántico, retórico) y la utilidad del enlace, y el segundo, la descripción física que analiza la dirección (interna, externa) y el medio hacia el cual se dirige (texto, imagen, etc.). Para una mejor definición de cada uno de los enlaces estos tipos se dividen en subcategorías. Los resultados convalidan la tipología y su aplicación a otras muestras de enlaces hipertextuales. Se nota que existe una gran tendencia de los periódicos a dar un lugar de privilegio a los enlaces semánticos, lo cual lleva, con mayor frecuencia, a un texto o a fuentes que provienen del mismo periódico.
L'utilisation de l'hypertexte permet d'établir des liens entre des textes ou les parties d'un même texte. On peut ainsi faire des relations directes entre les idées pour aider à construire le sens. Notre cerveau fonctionnant par associations d'éléments semblables, l'hypertexte aide le lecteur à forger son propre discours. II lui permet de consulter le supplément 
d'information qui lui convient à condition, bien sûr, qu'il y ait une réelle association entre ces liens, sinon l'hypertexte aura l'effet inverse, celui de déconstruire le sens et de semer la confusion. En naviguant d'un lien à l'autre, le lecteur moins familier avec le sujet peut aller chercher l'information qui lui manque, alors que le lecteur chevronné peut pousser plus loin sa propre analyse. II n'est plus emprisonné dans la structure linéaire du papier conçue pour un seul type de lecture.

Comme «[...]l'hypertexte devait permettre aux auteurs de relier des idées de manière plus riche et conforme au fonctionnement associatif de l'esprit humain " (Laufer et Scavetta 1992, 89), nous avons analysé, à l'aide d'une typologie de liens, les types de relations hypertextuelles d'articles de certains journaux qui publient une version électronique. Ce support documentaire a été choisi parce qu'il constitue une source importante d'information et pour voir du même coup si l'utilisation de l'hypertexte y est adéquate.

Dans le cadre de cette recherche, trois objectifs ont été établis. Le premier visait la création d'une typologie de liens hypertextuels adaptée au contexte des journaux électroniques. Le deuxième objectif validerait cette typologie à l'aide d'un échantillon de liens recueillis dans des articles de journaux. Un dernier objectif constaterait, à la lumière des résultats de l'analyse, si les journaux avaient su s'adapter aux possibilités discursives offertes par l'hypertexte à l'intérieur de leurs articles.

Pour atteindre ces objectifs, nous avons d'abord étudié ce que d'autres auteurs pensent de la version électronique des journaux et magazines. Nous avons aussi regardé ce que représente pour eux l'hypertexte avant de faire le tour des différentes typologies de liens déjà existantes.

\section{Revue de la littérature}

\section{Les journaux électroniques}

Le passage du papier à l'électronique offre plusieurs possibilités aux éditeurs de journaux. II n'y a plus de restriction en matière d'espace, donc plus de limite à la quantité d'information fournie sur un même sujet. De plus, l'hypertexte permet d'agencer l'information en de nombreu- ses manières (McAdams 1994). Le journaliste peut désormais tracer des chemins entre les informations et même les relier à ses propres sources, car l'hypertexte permet d'éliminer le «trou d'information» (causé par la limite d'espace du support papier) en reliant plusieurs informations au sujet traité. II peut donc superposer une ou plusieurs structures non linéaires au texte linéaire. (Laufer et Scavetta 1992; McAdams 1994 ; Lapham 1995)

Katz, Lapham, Lapointe et McAdams croient toutefois que la plupart des journaux n'ont pas su intégrer le meilleur des possibilités de l'hypertexte dans leur version électronique. Beaucoup n'offrent qu'une copie de la version papier alors que la lecture d'un hypertexte devrait être différente. L'importance de l'ordre des pages et la place des éléments d'information disparaissent, sauf pour la une (Katz 1994 ; McAdams 1994 ; Lapham 1995; Lapointe 1997). Certains journaux ont déjà décidé de produire un contenu original qui n'a parfois aucun lien avec le contenu de la version papier (Lapointe 1997). Les journaux doivent redéfinir leur mission pour offrir un produit adapté à ce nouveau support. Pour y parvenir, il faut revenir à la racine même du journalisme en produisant de l'information avant même de produire un journal. (Katz 1994 ; Lapham 1995)

\section{L'hypertexte}

Le concept de l'hypertexte, qui permet de sauter d'un document à l'autre selon des chemins préétablis, doit son invention à Vannevar Busch en 1945. Bush voulait concevoir une machine, le MEMEX, permettant d'automatiser la documentation et de relier les diverses informations selon plusieurs types d'associations. (Rhéaume 1993)

Pour les besoins de cette étude, nous nous sommes attardés aux possibilités de l'hypertexte sur le plan discursif. La définition retenue pour représenter le concept fut celle formulée par l'Office de la langue française (1997): "Présentation de l'information qui permet une lecture non linéaire grâce à la présence de liens sémantiques activables dans les documents. 》

La plus grande caractéristique de l'hypertexte est qu'il supporte la nonlinéarité d'un discours (Luiz dos Santos 199?; Rhéaume 1993; Clément 1995).
Cela n'est cependant pas un phénomène nouveau au sens où le support papier offrait déjà la possibilité de faire certains liens entre les portions d'un même texte ou d'autres textes, avec les notes de bas de page et les citations, mais il permet d'aller plus loin en dépassant les limites des formes déjà existantes (Luiz dos Santos 199?). L'hypertexte offre l'occasion au lecteur de pouvoir choisir son parcours de lecture en explorant les avenues qui l'intéressent le plus. Cette façon de lire transforme le lecteur en auteur puisqu'il peut former son propre discours (Luiz dos Santos 199?; Rhéaume 1993). II faut cependant faire attention à l'usage que l'on fait de l'hypertexte, car son utilisation massive et non pertinente risque de causer deux problèmes au lecteur, soit la désorientation et la surcharge cognitive. Les liens hypertextuels doivent donc être pertinents. (Rhéaume 1993)

\section{Les relations hypertextuelles}

Certains auteurs ont clairement tenté d'établir une typologie des liens hypertextuels que l'on retrouve dans le discours. Par exemple, Trigg (1983) a défini environ quatre-vingts types de liens qu'il divise en deux groupes principaux, soit les liens de commentaires qui critiquent ou commentent des éléments du discours et les liens normaux qui relient les références, les arguments, les solutions, les explications, les résumés et les exemples. Parunak (1991) distingue trois grandes classes de liens dont les deux premières s'inspirent de la linguistique discursive. Il y a d'abord les liens associatifs qui relient des nœuds d'informations similaires, puis les liens d'ensemble qui relient le tout à ses parties et les liens de révision qui relient les anciennes versions aux nouvelles. Quant à Thistlewaite (1997), il propose quatre types de liens: structurels reliant les objets au tout, référentiels, sémantiques et contingents qui présentent des liens ayant peu de similitude entre eux. Sans établir clairement de typologie, Rhéaume (1993) reconnaît au moins deux types de liens: référentiels (unis ou bidirectionnels) et organisationnels, qui s'apparentent aux liens structurels de Thistlewaite (1997). Baron et al. (1996) définissent deux grands types de liens: les 
liens organisationnels qui constituent des liens de direction à l'opposé de Thistlewaite et Rhéaume et les liens de contenu qui réunissent trois catégories: sémantiques (similaires, contraires, parties du tout), rhétoriques (définition, explication, illustration, résumé) et pragmatiques (exemple, avertissement, exercice).

\section{Méthodologie}

\section{Cadre d'analyse}

Faute d'avoir trouvé une typologie de liens hypertextuels parfaitement adaptée au contexte des articles de journaux, une nouvelle typologie a été établie en s'inspirant de celles de Baron et al. (1996) et de Thistlewaite (1997). De plus, quelques liens hypertextuels d'articles ont été observés pour servir de base à son élaboration. Elle est composée de deux groupes: description conceptuelle et description physique et divisée en quatre niveaux d'analyse différents.

\section{Description conceptuelle}

Les deux premiers niveaux d'analyse de la typologie se rapportent au sens et à l'utilité des liens. Ils sont les plus importants, car ils déterminent la fonction de l'hypertexte dans le discours.

\section{Premier niveau d'analyse: le sens du lien}

Pour ce niveau d'analyse, deux catégories de Baron et al. (1996), sémantique et rhétorique, ont été retenues. La catégorie pragmatique a été rejetée parce qu'elle ne s'appliquait pas au contexte.

\section{Première catégorie: les liens sémantiques}

La sémantique concerne ce qui est relatif au sens. La catégorie sémantique regroupe donc tout ce qui aide à comprendre le contexte de la situation présentée. Prenons, par exemple, le mot «éclipse "; un lien menant à sa définition dans un article présentant les dangers reliés à la prochaine éclipse solaire aiderait le lecteur à comprendre de quoi il s'agit, s'il ne connaît pas le phénomène.

\begin{tabular}{|c|c|c|c|c|c|c|c|c|c|}
\hline \multirow{2}{*}{ 悹 } & \multirow{2}{*}{ 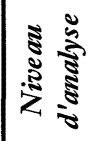 } & \multirow{2}{*}{ Catégorie } & \multirow{2}{*}{ Type } & \multicolumn{2}{|c|}{ fournal 1} & \multicolumn{2}{|c|}{ fournal 2} & \multicolumn{2}{|c|}{ fournal 3} \\
\hline & & & & Art.1 & Art. 2 & Art.1 & Art. 2 & Art. 1 & Art. 2 \\
\hline \multirow{16}{*}{ 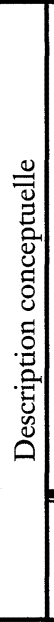 } & \multirow{13}{*}{ 抾 } & \multirow[t]{8}{*}{ Sémantique } & Général & & & & & & \\
\hline & & & Comparaison & & & & & & \\
\hline & & & \begin{tabular}{|l|} 
Contexte \\
\end{tabular} & & & & & & \\
\hline & & & Définition & & & & & & \\
\hline & & & \begin{tabular}{|l|} 
Exemple \\
\end{tabular} & & & & & & \\
\hline & & & Référence & & & & & & \\
\hline & & & Résumé & & & & & & \\
\hline & & & Autre & & & & & & \\
\hline & & \multirow[t]{5}{*}{ Rbétorique } & Cause & & & & & & \\
\hline & & & Commentaire & & & & & & \\
\hline & & & Comparaison & & & & & & \\
\hline & & & \begin{tabular}{|l|} 
Conséquence \\
\end{tabular} & & & & & & \\
\hline & & & Autre & & & & & & \\
\hline & \multirow{3}{*}{ | } & Pertinent & Pertinent & & & & & & \\
\hline & & \multirow[t]{2}{*}{ Redondant } & Différent & & & & & & \\
\hline & & & Identique & & & & & & \\
\hline & \multicolumn{3}{|c|}{ Total de liens } & & & & & & \\
\hline
\end{tabular}

Pour bien définir cette catégorie, il est nécessaire de la subdiviser en sept types:

- Le lien général est celui qui conduit le plus souvent à la page d'accueil d'un site externe au journal, sans être directement lié à un élément spécifique de l'article; il est plutôt relié au contexte d'ensemble. Ce type de lien peut désorienter le lecteur, car il ne le conduit pas vers un élément précis en fonction de l'article. II peut aussi s'agir d'un lien qui a un faible degré de relation avec le sujet. C'est le cas, par exemple, d'un article portant sur un écrasement d'avion au Japon qui serait relié à celui traitant d'un séisme ayant également eu lieu au Japon.

- Le lien de comparaison qui compare sans chercher à convaincre.

- Le lien de contexte qui renseigne le lecteur sur le sujet de l'article.

Le lien de définition explicite le sens d'un mot ou d'un événement. II peut aussi présenter le texte intégral d'un discours.

- Le lien d'illustration rassemble ce qui sert d'exemple à un texte: un article sur les dangers du tabac lié au cas d'un fumeur ayant un cancer des poumons.

- Le lien de référence qui renvoie à la source d'une citation pouvant en éclairer le contexte.

- Le lien de résumé qui conduit à un rappel des événements.

\section{Deuxième catégorie: les liens} rhétoriques

La rhétorique représente l'art de bien s'exprimer, spécialement dans un but de persuasion. II s'agit donc ici d'un lien qui amène des éléments nouveaux pour aider le lecteur à se former une opinion ou à le convaincre sur un sujet. En reprenant l'exemple de l'article sur les dangers potentiels de la prochaine éclipse solaire, un lien rhétorique conduirait à un article portant sur le nombre de personnes ayant déjà subi des dommages corporels à la suite d'autres éclipses.

La catégorie rhétorique se subdivise en quatre types:

- Le lien de cause qui unit la nouvelle à un article qui en explique les causes;

- Le lien de commentaire qui lie la nouvelle à un texte d'opinion ou à un forum de discussion;

Le lien de conséquence qui établit une relation entre la nouvelle et les effets qu'elle entraîne;

Le lien de comparaison qui met en parallèle deux événements semblables dans le but de convaincre. Par exemple, comparer deux tueries dans des écoles différentes pour convaincre de la présence de violence chez les jeunes. 
Dans la grille d'analyse, un type "autre» a été ajouté dans les catégories sémantique et rhétorique afin d'y réunir les liens hypertextes difficilement classables.

Il peut être parfois difficile de définir un lien dans un seul type parce qu'il peut correspondre à plusieurs. Dans le cadre de l'analyse, un lien a été classé dans un seul type en choisissant celui qui prédominait.

\section{Deuxième niveau d'analyse: l'utilité du lien}

Ce deuxième niveau est inspiré de la catégorie de liens contingents de Thistlewaite (1997). L'adjectif contingent est utilisé par opposition à nécessaire et désigne ce qui peut se produire ou non. II représente les liens superficiels qui ont un faible degré d'association entre eux. On retrouve souvent dans les articles des liens hypertextuels peu pertinents qui n'apportent rien à la compréhension pour le lecteur. II est donc intéressant d'analyser. l'utilité des liens à l'aide de deux catégories: pertinent et redondant.

\section{Première catégorie: les liens pertinents}

- Par opposition au lien redondant, les liens pertinents sont ceux qui peuvent être utiles au lecteur en apportant un éclairage nouveau à l'article de départ.

Par exemple, un article sur les successeurs potentiels du maire d'une ville pourrait traiter de la vie professionnelle des candidats ou des causes de sa démission. Cependant, un lien pourrait être pertinent sans aborder directement le sujet de la démission; par exemple, un article pourrait retracer la carrière professionnelle du maire, par contre, un lien sur son voyage en Chine serait hors du sujet et il devrait être classé sous redondant différent.

\section{Deuxième catégorie: les liens redondants}

- Le lien identique reprend le même contenu sans y apporter d'élément nouveau et différent; il a un faible degré de relation sémantique.

- Le lien différent est aussi qualifié de redondant, non pas au sens de répétition, mais plutôt au sens de superflu, car il dirige le lecteur vers un autre sujet.

Tableau 2. Typologie de liens hypertextuels: description physique

\begin{tabular}{|c|c|c|c|c|c|c|c|c|c|}
\hline \multirow{2}{*}{ है } & \multirow{2}{*}{ 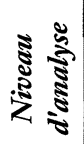 } & \multirow{2}{*}{ Catégorie } & \multirow{2}{*}{ Type } & \multicolumn{2}{|c|}{ Fournal 1} & \multicolumn{2}{|c|}{ Fournal 2} & \multicolumn{2}{|c|}{ fournal 3} \\
\hline & & & & Art.1 & Art.2 & Art.1 & Art. 2 & Art.1 & Art.2 \\
\hline \multirow{10}{*}{ 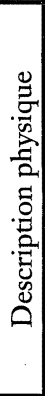 } & \multirow{4}{*}{. } & \multirow[t]{2}{*}{ Externe } & Page d'accueil & & & & & & \\
\hline & & & Page spécifique & & & & & & \\
\hline & & \multirow[t]{2}{*}{ Interne } & Autre texte & & & & & & \\
\hline & & & Même texte & & & & & & \\
\hline & \multirow{6}{*}{ 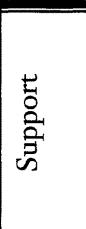 } & & Formulaire & & & & & & \\
\hline & & & Image & & & & & & \\
\hline & & & Lien & & & & & & \\
\hline & & & Son & & & & & & \\
\hline & & & Texte & & & & & & \\
\hline & & & Vidéo & & & & & & \\
\hline & \multicolumn{3}{|c|}{ Total de liens } & & & & & & \\
\hline
\end{tabular}

\section{Description physique}

Les deux autres niveaux d'analyse, soit la direction et le support, ne font qu'ajouter des critères de classification secondaires de description physique du lien. Ils offrent toutefois la possibilité d'établir des corrélations avec les liens conceptuels et de faire ressortir les tendances des journaux.

\section{Troisième niveau d'analyse: la direction du lien}

Ce niveau est subdivisé en deux catégories de liens: externe et interne.

\section{Première catégorie: les liens externes}

Le lien externe dirige hors du site, en l'occurrence du journal.

Cette catégorie se divise en deux types:

la page d'accueil,

la page spécifique, celle-ci offrant de l'information liée au sujet de l'article de façon directe: un texte sur la cigarette lié à une page présentant les nouvelles lois.

\section{Deuxième catégorie: les liens internes}

Les liens internes sont ceux qui dirigent vers une unité d'information à l'inté- rieur même de l'article ou dans un article différent appartenant au journal.

Cette catégorie contient deux types:

- le lien vers le même texte;

vers un autre texte.

\section{Quatrième niveau d'analyse: le support du lien}

Ce dernier niveau d'analyse sert à décrire vers quel type de support dirige le lien.

Il comporte six types de liens:

le formulaire,

: l'image,

- le lien vers une liste de liens hypertextuels sans aucun autre contenu,

- le texte qui conduit vers un texte continu,

le son,

vidéo.

La typologie définie permet donc de classer un même lien dans chacun des quatre niveaux d'analyse. Par exemple, on peut avoir un lien rhétorique de cause, pertinent, interne, qui renvoie à un autre texte contenant un texte.

\section{Échantillon}

Pour valider cette nouvelle typologie, un échantillon de 170 liens hypertextuels tirés d'articles de neuf journaux quotidiens et deux mensuels ont été analysés (voir le tableau 4). II importait d'avoir un nombre suffisant de journaux et de liens 
Tableau 3. Résultats de l'analyse

\begin{tabular}{|c|c|c|c|c|c|}
\hline है। & 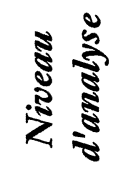 & Catégorie & Type & $\begin{array}{c}\text { Total } \\
\text { de liens }\end{array}$ & $\%$ de liens \\
\hline \multirow{18}{*}{ 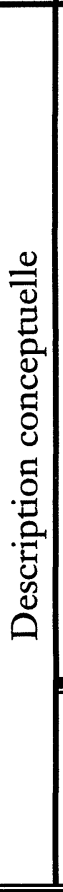 } & \multirow{14}{*}{ 离 } & \multirow[t]{8}{*}{ Sémantique } & Général & 45 & $26 \%$ \\
\hline & & & Comparaison & 0 & $0 \%$ \\
\hline & & & Contexte & 40 & $23 \%$ \\
\hline & & & Définition & 24 & $14 \%$ \\
\hline & & & Exemple & 1 & $1 \%$ \\
\hline & & & Référence & 6 & $4 \%$ \\
\hline & & & Résumé & 3 & $2 \%$ \\
\hline & & & Autre & 4 & $2 \%$ \\
\hline & & \multirow[t]{6}{*}{ Rbétorique } & Cause & 11 & $6 \%$ \\
\hline & & & Commentaire & 12 & $7 \%$ \\
\hline & & & Comparaison & 6 & $4 \%$ \\
\hline & & & Conséquence & 15 & $9 \%$ \\
\hline & & & Autre & 3 & $2 \%$ \\
\hline & & & Total & 170 & $100 \%$ \\
\hline & \multirow{4}{*}{ 营 } & Pertinent & Pertinent & 144 & $85 \%$ \\
\hline & & Redondant & Différent & 19 & $11 \%$ \\
\hline & & & Identique & 7 & $4 \%$ \\
\hline & & & Total & 170 & $100 \%$ \\
\hline \multirow{11}{*}{ 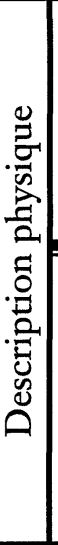 } & \multirow{5}{*}{ 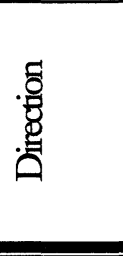 } & \multirow[t]{2}{*}{ Externe } & Page d'accueil & 28 & $16 \%$ \\
\hline & & & Page spécifique & 17 & $10 \%$ \\
\hline & & \multirow[t]{2}{*}{ Interne } & Autre texte & 119 & $70 \%$ \\
\hline & & & Même texte & 6 & $4 \%$ \\
\hline & & & Total & 170 & $100 \%$ \\
\hline & \multirow{6}{*}{ 范 } & & Formulaire & 4 & $2 \%$ \\
\hline & & & Image & 10 & $6 \%$ \\
\hline & & & Lien & 22 & $13 \%$ \\
\hline & & & Son & 0 & $0 \%$ \\
\hline & & & Texte & 134 & $79 \%$ \\
\hline & & & Vidéo & 0 & $0 \%$ \\
\hline \multicolumn{4}{|c|}{ Total de liens } & 170 & $100 \%$ \\
\hline
\end{tabular}

afin de réunir plusieurs types de relations. Ainsi les liens hypertextuels de deux ou trois articles ont été retenus pour faire partie de l'échantillon. Dans le cas des mensuels, les liens d'un dossier complet ont été retenus. Seuls les liens reliant des éléments d'information se trouvant à l'intérieur ou en marge de l'article d'ori- gine se sont retrouvés dans l'échantillon. Les liens organisationnels qui réfèrent à la structure même du document n'ont pas été gardés. II fallait donc établir les types de relations entre deux unités d'information reliées. Les résultats ont ensuite servi à valider la typologie de liens hypertextuels utilisée et voir son application à d'autres échantillons d'articles de journaux ou peut-être même à d'autres types de documents.

L'échantillon a été constitué à l'aide d'outils de recherche d'Internet. Pour être choisis, les journaux devaient être plutôt de portée générale. Ces journaux devaient également contenir plus d'un article ayant des liens hypertextes menant à des informations complémentaires ou à des articles antérieurs sur le même sujet.

\section{Résultats de l'analyse}

Le tableau 3 présente la compilation des résultats de l'analyse des liens. Les quatre premières colonnes montrent la typologie de liens hypertextuels. La colonne suivante "Total de liens" représente le nombre total de liens de l'échantillon pour chacun des éléments de la typologie. Finalement, la colonne "Pourcentage de liens » indique le pourcentage correspondant à chacun des types par rapport aux 170 liens évalués.

\section{Description conceptuelle}

Au niveau d'analyse du sens, près de trois liens analysés sur quatre sont de catégorie sémantique. Le plus grand pourcentage, $26 \%$, est de type sémantique général, suivi du type sémantique de contexte avec $23 \%$ et du type sémantique de définition, $14 \%$. Ce sont ces trois types que l'on retrouve le plus souvent dans les articles. Seul le type sémantique de comparaison obtient $0 \%$, tandis que les autres types de liens sémantiques représentent à peine $9 \%$ de l'ensemble des liens. Du côté de la catégorie rhétorique, il n'y a pas de différence marquée entre les types. La rhétorique de conséquence obtient le plus grand nombre de liens avec $9 \%$, suivi de près par la rhétorique de commentaire, $7 \%$ et la rhétorique de cause avec $6 \%$.

Le deuxième niveau d'analyse, l'utilité du lien, propose un grand nombre du type de liens pertinents, soit $85 \%$. Ce résultat doit cependant être nuancé, car il présente une fausse vision de l'utilité des liens. II en sera discuté plus en détails dans la section suivante. Notons que peu de liens se sont avérés de type identique, soit $4 \%$. En général, ces liens mènent exactement au même texte. 


\section{Description physique}

À l'intérieur du troisième niveau d'analyse des liens de la typologie, direction, près de trois liens sur quatre se situent dans la catégorie interne, soit $74 \%$. Sous la catégorie externe, il y a peu de différence entre les liens dirigeant vers une page d'accueil, $16 \%$ et $10 \%$ vers une page spécifique au sujet. La section suivante établira cependant une nuance entre ces deux types de liens.

Finalement, le niveau d'analyse support montre que le type de lien le plus répandu, $79 \%$, est le texte, ce qui était prévisible. Il est aussi intéressant de noter que $13 \%$ des liens renvoient vers une page contenant une liste de liens et que certains journaux font des liens vers des images dans une proportion de $6 \%$.

\section{Discussion et conclusion}

\section{Validation de la typologie}

Les objectifs principaux visaient la création et la validation d'une typologie de liens hypertextuels. À la lumière des résultats, évaluons l'utilité et la pertinence de chacun des types de liens de cette typologie en fonction d'une utilisation future.

Dans l'ensemble, les niveaux d'analyse et les catégories étaient suffisamment bien définis pour l'échantillon analysé. Bien que le classement des liens ait posé peu de problèmes, certaines catégories et certains types devraient être redéfinis et modifiés légèrement.

\section{Description conceptuelle}

\section{Premier niveau d'analyse: le sens du lien}

Les subdivisions définies au départ se sont toutes avérées pertinentes même si on ne retrouvait pas de liens sous chacune. C'est le cas des types sémantique illustration et sémantique comparaison qui n'ont recueilli aucun lien. Pourtant, ils pourraient être utiles dans les cas où l'on se servirait d'exemples ou de comparaisons pour illustrer une idée. Le type sémantique résumé, qui ne représente que $2 \%$ des liens, demeure aussi très pertinent. Les liens qu'on y retrouve situent bien le contexte d'un article en rappelant les événements.

À l'inverse, on trouve des types où le nombre de liens semble trop grand. C'est le cas de sémantique général qui regroupe plus d'un lien sur quatre dans l'échantillon étudié. II devrait être mieux circonscrit afin d'éviter qu'un nombre élevé de liens s'y retrouve. À l'origine, il ne devait contenir que les liens traitant du sujet de l'article mais largement, comme par exemple la page d'accueil de l'Afrique du Sud qui est reliée au dossier du mois du Courrier international (mars 1998) : cet article n'était pas précisément lié sémantiquement au dossier mais il l'était globalement. Lors de l'analyse de l'échantillon, un autre type de relation s'est aussi retrouvé sous l'appellation sémantique général: il s'agit des liens ayant une faible relation sémantique avec l'article d'origine. Ainsi, dans l'article du Boston Globe (14 mars 1998) où l'on parle de la démission de Joseph Kennedy, on trouve un lien vers une page présentant des nouvelles de la région du nord-est des États-Unis. Le lien sémantique entre ces articles est faible et ne repose que sur le lieu géographique. Les liens redondants identiques y ont aussi été classés, car ils ne convenaient à aucun autre type de cette catégorie. II serait donc préférable de diviser ce type en deux pour distinguer les liens "faibles » et les liens véritablement "généraux".

Le type sémantique référence visait, d'abord et avant tout, les articles comportant des citations faisant référence aux auteurs. À cela s'est ajouté, lors de l'analyse, le lien vers le courrier électronique des auteurs des articles.

La case sémantique autre de la grille d'analyse, nous a permis d'identifier deux nouveaux types ainsi qu'une nouvelle catégorie. Le premier type est sémantique d'explication; il regroupe les liens où l'on reprend des idées - un peu comme dans le type sémantique résumé - non pas pour être plus concis, mais plutôt pour expliquer et approfondir une facette d'un sujet. C'est dans le USA Today (2 avril 1998) que ce type de lien est apparu. Ce journal ajoute des liens hypertextuels au résumé de l'article, plutôt qu'à l'article de fond. La catégorie pragmatique, que l'on retrouvait dans la typologie de Baron et al. (1996), a aussi été ajoutée. Elle avait été abandonnée parce qu'elle visait surtout les exercices pratiques. Or, un article du New Jersey Online (2 avril 1998) offrait un lien vers un formulaire de recherche. Le lecteur était donc amené à agir, à poser une action, d'où l'ajout de pragmatique. Puisque Internet permet l'interactivité, ce lien est appelé à être de plus en plus présent. Sous cette nouvelle catégorie se retrouve donc un type de lien du même nom, soit le type pragmatique.

En ce qui concerne la catégorie rhétorique, les résultats en valident l'utilité. La case rhétorique autre a permis de définir un nouveau type de lien, soit la rhétorique générique qui regroupe les liens menant à des articles couvrant un sujet plus vaste, mais englobant celui de l'article de départ. Ainsi, dans le New York Times (31 mars 1998), un article sur l'exécution d'une condamnée à mort présente un lien menant vers un second article sur la peine de mort. Celui-ci dépasse le sujet - la condamnation à mort de cette femme puisqu'il remet en cause l'idée même de la peine de mort. Il s'agit donc d'un lien à caractère plus générique. L'ajout de ce type de lien entraîne aussi l'ajout de son opposé, la rhétorique d'exemple. Bien qu'aucun lien de ce type n'ait été analysé, son ajout est cependant nécessaire, car l'inverse est aussi possible, c'est-à-dire un article traitant de la peine de mort de façon générale peut mener au cas particulier d'un individu.

\section{Deuxième niveau d'analyse : l'utilité du lien}

Le classement des liens selon le niveau d'analyse de l'utilité s'est avéré difficile parce qu'il repose sur des facteurs subjectifs. Cela explique qu'on retrouve $85 \%$ des liens sous la catégorie pertinent dans le tableau 3. Avant de commencer, il semblait facile et évident de juger de la pertinence d'un lien, mais à l'usage, il est clairement apparu que des critères auraient dû être définis, mais il était alors trop tard pour y revenir. Par exemple, il était difficile de juger de l'utilité d'un lien menant à la page d'accueil d'un site vaste classé sous la catégorie pertinent. Par défaut, un tel lien se retrouvait sous pertinent, alors qu'il aurait probablement dû se trouver sous redondant différent, puisque la relation entre les deux textes n'apparaissait pas clairement. Pour tenter d'éviter le piège de la subjectivité, il serait important d'établir des critères précis 
d'évaluation, ce qui n'avait pas été fait au préalable avant l'expérimentation. Lors de l'analyse, l'absence de critères a rendu le classement difficile comme ce fut le cas pour les images.

Les liens de commentaire causaient aussi problème. Comment juger de la pertinence d'un commentaire? En considérant que toutes les opinions se valent et qu'elles apportent un point de vue sur un sujet, elles ont donc toutes été considérées pertinentes, mais est-ce vraiment le cas?

Un dernier point reste à discuter, celui des liens qui sont pertinents (parce qu'ils permettent d'approfondir un sujet), mais qui désorientent le lecteur par leur envergure. Par exemple, un article sur la situation politique en Afrique du Sud qui renvoie au site officiel de ce pays pour permettre au lecteur d'en augmenter la connaissance. Se retrouver devant la page d'accueil d'un pareil site désoriente le lecteur, car ce lien risque d'apporter plus de questions que de réponses. Dans ces conditions, malgré le potentiel d'information pertinente qui s'y trouve, ce lien a un effet contraire à celui recherché et devrait être classé dans le type redondant différent.

Même si plusieurs problèmes sont survenus lors de l'analyse de l'utilité, ce niveau est nécessaire, car c'est lui qui permet de porter un jugement sur la mauvaise utilisation de l'hypertexte. Pour simplifier l'analyse, un lien devrait être considéré comme pertinent s'il facilite la compréhension du sujet ou s'il aide le lecteur à se former une opinion, sans le désorienter. $\mathrm{Ce}$ critère, bien qu'un peu vague, constitue un point de départ essentiel malgré une part de subjectivité qu'il est difficile d'éviter.

\section{Description physique}

À prime abord, il peut apparaître peu pertinent d'analyser les liens direction et support. Ces niveaux d'analyse sont cependant utiles pour déterminer les tendances adoptées par les journaux, mais surtout dans l'analyse des corrélations existant entre les catégories conceptuelle et physique.

\section{Troisième niveau d'analyse : la direction du lien}

L'analyse de la direction du lien a posé peu de problèmes. Le seul élément de discussion concerne la catégorie externe. L'examen de l'échantillon a démontré qu'il faudrait modifier les types page d'accueil et page spécifique (général et spécifique), car une page d'accueil présente parfois de l'information spécifique: par exemple, la page d'accueil du journal Africa News, liée à l'article du Chicago Tribune (27 mars 1998) traitant du voyage de Bill Clinton en Afrique du Sud, est reliée spécifiquement au sujet, car on y retrouve des articles sur le voyage en première page. Le type général engloberait alors les liens vers de l'information générale concernant l'article.

Le dernier type de lien, support, fut le plus facile à analyser et il n'exige aucune correction.

\section{Les journaux et l'hypertexte}

En ce qui a trait à l'objectif concernant l'adaptation des journaux aux possibilités offertes par l'hypertexte, plusieurs points méritent d'être soulignés.

La majorité des journaux analysés dirigent vers des liens extérieurs au texte, sous la forme d'une liste accompagnant l'article. Très peu d'articles disposent de liens à l'intérieur même du texte sur des mots précis. Notons aussi que les journaux présentent habituellement leurs liens hypertextuels de la même façon dans chacun de leurs articles. Ils sont aussi fidèles dans la direction qu'ils donnent aux liens, certains font des liens externes et internes tandis que d'autres ne font que des liens internes. Finalement, on observe que les journaux n'offrent pas de liens hypertextuels dans tous leurs articles. Ce ne sont souvent que les sujets principaux, qui ont une bonne couverture s'étalant sur plusieurs jours, qui en possèdent.

Une facette intéressante du journal électronique est qu'il permet de lier facilement des articles touchant le même sujet sur une période de plusieurs jours, voire de plusieurs mois. Le lecteur peut suivre plus aisément un dossier, car il a devant lui tous les éléments nécessaires à sa compréhension. Le cerveau fonctionnant par associations d'idées, l'hypertexte permet a priori une meilleure compréhension d'un événement que la version papier des journaux.

Plus intéressant encore, le journal électronique permet de relier des événe- ments distincts mais similaires. C'était le cas, entre autre, du Washington Post (27 mars 1998) qui traitait d'une tuerie par des jeunes dans une école de l'Arkansas; l'article était relié à deux autres histoires du même genre, de jeunes ayant eu recours à la violence. Plusieurs facettes du sujet sont montrées afin de mieux en saisir les enjeux.

L'analyse de l'échantillon fait ressortir l'accent mis par les journaux sur le côté sémantique de l'hypertexte. En effet, la majorité utilise les liens informatifs plutôt qu'argumentatifs. L'hypertexte offre pourtant de superbes possibilités rhétoriques bien que le travail de recherche soit plus complexe et demande une indexation approfondie des articles.

\section{Études futures}

La typologie définie permet d'analyser les liens hypertextuels sur deux niveaux de profondeur. En effet, on peut se limiter au premier niveau, sémantique ou rhétorique, ou pousser plus loin l'examen et définir le type de lien sémantique dont il s'agit. II serait donc intéressant d'appliquer cette typologie de liens hypertextuels à d'autres styles de textes que l'article de journal pour voir si elle peut convenir. II serait aussi intéressant d'analyser un échantillon plus vaste de liens pour faire ressortir les tendances des différents journaux et pour analyser les relations entre les catégories afin de savoir s'il existe une corrélation entre elles. Ces remarques pourraient faire l'objet d'une nouvelle étude visant, cette fois, l'analyse statistique des résultats, puisque la typologie est déjà définie. Cependant, rappelons qu'il faudra établir des critères de pertinence spécifiquement liés au contexte pour analyser l'utilité du lien.

Dans une perspective plus large, cette étude ouvre la porte à l'analyse du discours hypertextuel. Offrant des possibilités d'agencement de l'information inédites, ce nouveau type de document demande l'adaptation de nos méthodes d'analyse puisqu'il est appelé à changer nos habitudes de lecture et de nos aptitudes à la compréhension. 


\section{Tableau 4. Journaux}

The Boston Globe Online (deux articles du 14 mars 1998)

url:http ://www.boston.com/globe

Chicago Tribune (deux articles du 27 mars 1998) url: http://www.chicago.tribune.com

Courrier International (dossier de mars 1998) url: http ://www.expansion.tm.fr/courrier/

Le monde diplomatique (dossier de mars 1998) url: http://www.monde-diplomatique.fr

New Jersey Online (deux articles du 2 avril 1998) url: http://www.nj.com

The New York Times (deux articles du 27 et du 31 mars 1998) url: http://www.nytimes.com

San Jose Mercury News (deux articles du 13 mars 1998) url: http ://www.sjmercury.com/

Time Magazine Daily (trois articles du 31 mars 1998) url: http://www.pathfinder.com/time/

U.S. News Online (deux articles du 2 avril 1998) url: http://www.usnews.com/usnews/ home.htm

USA Today (trois articles du 21 février et du 2 avril 1998) url: http ://www.usatoday.com

The Washington Post (deux articles du 23 et du 27 mars 1998)

url: http://washingtonpost.com

\section{Sources consultées}

Baron, Lisa et al. 1996. Labeled, typed links as cues when reading hypertext documents. Journal of the American Society for Information Science 47 (12): 896-908.

Clément, Jean. 1995. Du texte à l'hypertexte: vers une épistémologie de la discursivité hypertextuelle. Acheronta n 2 : http://www.psiconet.com/ acheronta/acheronta2/dutextel.htm.

Deshaies, Bruno. 1992. Méthodologie de la recherche en sciences humaines. Laval: Éditions Beauchemin. $400 \mathrm{p}$.

Katz, Jon. 1994. Online or not, newspapers suck. Wired 2 (9): http://www. wired.com/wired/2.09/departments/electrosphere/news.suck.html.

Lapham, Chris. 1995. The evolution of the newspaper of the future. CMC Magazine (juillet): http://sunsite.unc.edu/cmc/mag/1995/jul/lapham.html.

Lapointe, Pascal. 1997. Les journaux sur le Web: construire pour l'avenir? La Presse (15 octobre): B 10.

Laufer, Roger et Domenico Scavetta. 1992. Texte, hypertexte, hypermédia. Que sais-je? n²629. Paris: Presses Universitaires de France. $125 \mathrm{p}$.

Luiz dos Santos, Alckmar. 199?. À propos d'une textualité Informatisée. http://www.cce.ufsc.br/ alckmar/texto6.html
McAdams, Melinda. 1994. Driving a newspaper on the data highway. http://www.well.com/user/mmca dams/online.newspapers.html.

Office de langue française. 1997. Vocabulaire d'Internet plus. http://www.olf.gouv.qc.ca/service/pages/internet2.html.

Parunak, H. Van Dike. 1991. Ordering the information graph. In Hypertext/Hypermedia handbook New York: Intertext Publications, McGraw-Hill Publishing Company, p. 299-325.

Rhéaume, Jacques. 1993. Les hypertextes et les hypermédias. L'informatique pédagogique 1 (2): http ://www.fse.ulaval.ca/fac/ten/reveduc/html/ vol1/no2/heth.html.

Thistlewaite, Paul. 1997. Automatic construction and management of large open webs. Information Processing \& Management 33 (2): 161-173.

Trigg, Randall. 1983. A taxonomy of link types. In $A$ Network-based approach to text handling for the online scientific community. http://www.parc.xerox.com/spl/members/trigg/thesis/thesischap4.html.

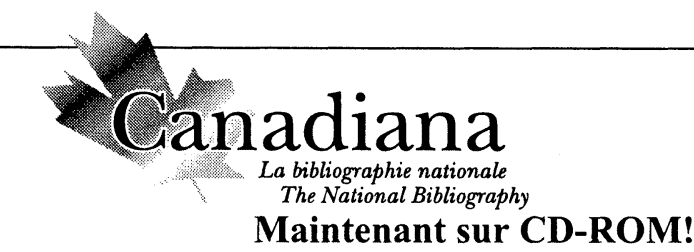

\section{Maintenant sur CD-ROM! \\ Un nouveau produit de la Bibliothèque nationale du Canada!}

Couvrant les années 1973 à 1997, Canadiana répertorie et décrit une vaste gamme de documents produits au Canada. Le cédérom, avec plus de 1,2 million de notices, comprend également Canadiana: Vedettes d'autorité et Carto-Canadiana, ainsi que les manuels sur le MARC canadien.

Exploitez les grandes possibilités du cédérom Canadiana qui facilite la recherche et qui permet le catalogage dérivé et la vérification de renseignements.

Pour une démonstration : hHp://www.nlc-bnc.ca/canadiana/

Prix : $129,95 \$(139,05 \$$ en incluant la TPS de $7 \%)$

No de cat. : SN2-2/1997-MRC

Pour placer une commande par:

Courrier : Les Éditions du gouvernement du Canada, Ottawa (Ontario), K1A 0S9, Téléphone : (819) 956-4800, Télécopieur :

(819) 994-1498, Internet : http://publications.pwgsc.gc.ca

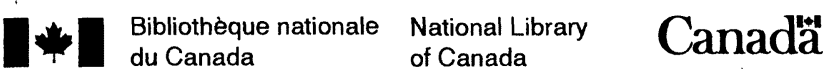

